\title{
Model for Aerobic Growth of Bacillus Amyloliquefaciens in Processed \\ Soy Sauce under Various Conditions of Temperature, Initial Dry Cell Mass and Ethanol Concentration
}

\author{
Yoshio MAKINO \\ Graduate School of Agricultural and Life Sciences, The University of Tokyo, 1-1-1 Yayoi, Bunkyo-ku, Tokyo 113-8657, Japan
}

Received October 18, 2004; Accepted January 22, 2005

\begin{abstract}
Construction of a predictive model for hygenic management of liquid foods was attempted by investigating the effects and interactions of storage conditions on the aerobic growth kinetics of Bacillus amyloliquefaciens in a soup product. The Gompertz curves were generated from the experimental data obtained under three temperatures $(298$ to $310 \mathrm{~K})$ in combination with four levels of inoculation $\left(5.32 \times 10^{-3}\right.$ to $\left.5.32 \times 10^{-6} \mathrm{~g} \cdot \mathrm{L}^{-1}\right)$ and five levels of ethanol concentration $\left(2.7\right.$ to $\left.4.7 \mathrm{~g} \cdot \mathrm{L}^{-1}\right)$. The microbial growth kinetic analysis disclosed that ethanol was effective for prolonging lag phase duration of the growth in a processed soy sauce. The Gompertz function in combination with nonlinear equations generated by stepwise regression analysis was suitable for predicting the growth of organisms. The lag phase duration was about $18 \mathrm{~h}$ longer than the quality guarantee period of a soup product containing a perceptible amount of contaminant organisms.
\end{abstract}

Keywords: liquid seasoning, genus Bacillus, predictive microbiology, spoilage, Gompertz function, multiple regression analysis

\section{Introduction}

Soups for noodles such as ramen and soba, or for one-pot dishes are prepared in individual restaurants by mixing various ingredients. Recently, products including extracts from natural ingredients for which the taste approaches that of restaurants have been distributed to the market by soy sauce makers and others. In the popular oriental market, particularly that of the processed soup products including soy sauce, natural extracts and the other ingredients similar amounts of umami taste substances like mono sodium glutamate, inosinic acid and others are added to the soup prepared by cooks in restaurants. However, the soup products are easily spoiled by microorganism contamination from soy sauce, as the deficiency of acidity, water activity and ethanol fail to depress the growth of the contaminants. Therefore, quality of the products has to be monitored continuously from a safety viewpoint.

Predictive food microbiology brings together the disciplines of food microbiology, engineering and statistics to provide useful predictions about microbial behavior in food systems. The potential of predictive microbiology has attracted considerable research interest since the 1980 s with funding of the United Kingdom and United States of America. Research projects of the Ministry of Agriculture, Fisheries and Food in the UK, and the United

E-mail: amakino@mail.ecc.u-tokyo.ac.jp
States Department of Agriculture in USA have borne fruit in the forms of 'Food Micromodel' and 'Pathogen Modeling Program', respectively (Ross and McMeekin 1994). Since the existing programs cannot be applied to the growth prediction of genus Bacillus as main contaminants in soups, except for toxin-producing species like $B$. cereus, a model suitable for the spoilage of soup products is needed. Many models to predict the growth of microorganisms have been proposed to date. Buchanan and Phillips (1990), Zaika et al. (1992), McClure et al. (1994) and Bhaduri et al. (1995) expressed microbial growth using the Gompertz equation with parameters calculated by second order response surface models as functions of temperature, contents and so on. Schoolfield et al. (1981) and Ratkowsky et al. $(1982,1983)$ proposed the Eyring equation and empirical square-root models to describe the temperature dependence of microbial growth, respective1y. The later models have been employed by many microbiologists.

Since an infinite number of seasoning combinations for soups exist, it is very difficult to model relationships between the growth of contaminants and the content of soups. Therefore, we attempted to construct a predictive model for the growth of a heatstable bacterium in a soup product for which the content had been determined. In transport and storage processes, an initial amount of contaminant and atmospheric temperature affect the spoilage of the soup product, except for the contents. 
Table 1. Contents in a soup product used for the cultivation of Bacillus amyloliquefaciens KP-991 strain.

\begin{tabular}{cccccc}
\hline $\begin{array}{c}\text { Total } \\
\text { nitrogen }\end{array}$ & $\begin{array}{c}\text { Sodium } \\
\text { chloride }^{*}\end{array}$ & $\begin{array}{c}\text { Direct reducing } \\
\text { sugar }^{*}\end{array}$ & Ethanol $^{*}$ & PH & Water activity \\
\hline 3.40 & 33.8 & 23.5 & 2.7 & 5.25 & 0.96 \\
\hline${ }^{*} \mathrm{~g} \cdot \mathrm{L}^{-1}$ & & & & &
\end{tabular}

Moreover, the effect of ethanol on the preservation of soup is also investigated. The proposed method will be applied to the prediction of growth by changing the parameter values in the model, even for food products with contents different from those in this study.

\section{Materials and Methods}

Strain and inoculum preparation The heatstable aerobic bacterium KP-991 strain isolated from raw soy sauce produced in Uchinomi (Kagawa prefecture, Japan), which was identified as Bacillus amyloliquefaciens (Fukumoto) Priest et al. by Makino and Fujisawa (2001), was used. A platinum loop was used to transfer organisms from nutrient agar slant to a small amount of nutrient broth (Claus and Berkeley 1986). The cell suspension was added to 50 $\mathrm{mL}$ of nutrient broth in a $250 \mathrm{~mL}$ shaking flask. A precultured cell suspension was prepared by shaking (velocity $120 \mathrm{rpm}$, amplitude $3 \mathrm{~cm}$ ) the flask for $17 \mathrm{~h}$ at $30^{\circ} \mathrm{C}$. In this study, vegetative cells were used as inoculum instead of spores, which are not distinguished from vegetative ones by spectrophotometry. Microbial inoculums were prepared by 10 -fold dilutions of harvested cell suspensions at four levels of dry cell mass concentrations $(5.32 \times$ $10^{-3}, 5.32 \times 10^{-4}, 5.32 \times 10^{-5}$ and $5.32 \times 10^{-6} \mathrm{~g} \cdot \mathrm{L}^{-1}$ ) using the soup product for soba noodle purchased from a soy sauce maker, which was sterilized by filtration with a $4.5 \times 10^{-4}$ mm membrane (Advantec MFS. INC., Tokyo Japan) before dilution. Haga and Endo (1971) reported that the number of spores contained in soy sauce products was in the range of $10^{3}$ to $10^{9} \mathrm{CFU} \cdot \mathrm{L}^{-1}$ and transformed into $2.5 \times$ $10^{2}$ to $2.5 \times 10^{8} \mathrm{CFU} \cdot \mathrm{L}^{-1}$ when the products were diluted four-fold with water, including natural extracts for soup manufacturing. The range of inoculum size in this study was in the range of $10^{3}$ to $10^{6} \mathrm{CFU} \cdot \mathrm{L}^{-1}$, which is included in the reported contamination levels. Terasawa et al. (1993) and Ohtomi et al. (1992) inoculated $10^{5} \mathrm{CFU} \cdot \mathrm{L}^{-1}$ genus Bacillus spores and $10^{6} \mathrm{CFU} \cdot \mathrm{L}^{-1}$ genus Bacillus vegetative cells to soup for pasteurization and growth tests, respectively. Therefore, the inoculum sizes in this study were selected. Dry cell mass in the harvested crude suspension $\left(\mathrm{g} \cdot \mathrm{L}^{-1}\right)$ was determined by spectrophotometry at O.D. $660 \mathrm{~nm}$ with a prepared calibration curve. The contents of the soup product examined in this study are presented in Table 1. Total nitrogen, $\mathrm{NaCl}$, direct reducing sugar, ethanol and $\mathrm{pH}$ in the soup were analyzed by the micro-Kjeldahl method, the Mohr method, 3,5-dinitrosalicylic acid test, gas chromatography and glass electrode test, respectively. Water activity at $25^{\circ} \mathrm{C}$ was determined with an EZ-100 water activity meter (measurable range 0.30-0.98, minimum value 0.02) (Freund Industrial Co., Ltd., Tokyo, Japan).

Growth media preparation The subjected soup product was supplemented with $0,0.5,1.0,1.5$ or $2.0 \mathrm{~g} \cdot \mathrm{L}^{-1}$ ethanol to provide total concentrations of 2.7, 3.2, 3.7, 4.2 or $4.7 \mathrm{~g} \cdot \mathrm{L}^{-1}$ ethanol, respectively.

Growth monitoring A $0.1-\mathrm{mL}$ inoculum was added to a $16.5 \mathrm{~mm} \phi \times 165 \mathrm{~mm}$ test tube containing $9.9 \mathrm{~mL}$ of growth media. Then, the inoculated media were stored quietly under aerobic conditions with the top of tube being covered with an aluminum cap. $\mathrm{As}_{2}$ in the air was continuously supplied to the media through the slit between the walls of the tube and the cap, growth condition for the inoculum in this study were better than in actual products of which the bottle is completely closed. However, the results obtained in this study may be adaptable for practical use because the predicted spoilage is estimated to be earlier than the actual one. Selection of a facultatively anaerobic bacterium as an inoculum may be effective for reducing the margin of growth rates. Cultivation was performed to assess the effects of temperature (298, 303 and $310 \mathrm{~K})$ in combination with four levels of inoculated dry cell mass and five levels of ethanol concentration. The growths of organisms were monitored by measuring O.D. $660 \mathrm{~nm}$ at appropriate intervals using a V530iRM spectrophotometer (JASCO, Tokyo, Japan). The test tubes were gently shaken with a vortex mixer before each measurement to ensure a uniform suspension. All combinations were replicated three times. Net absorbance was determined by subtracting values of O.D. of soup and test tube wall from a whole value.

Kinetic analysis of growth Makino and Fujisawa (2001) reported that $B$. amyloliquefaciens grew sigmoidally in processed soy sauces. Several sigmoidal functions are effective for approximating the growth curves (Zwietering et al. 1990). Growth curves were generated from the experimental data obtained in the present study using the Gompertz function (Eq. 1) which has been most employed (Ross and McMeekin 1994) in conjection with SigmaProt ${ }^{\circledR}$ ver.5 (SPSS Inc., Chicago, Illinois, USA), a nonlinear least square fitting program that employs the MarquardtLevenberg algorithm.

$$
N_{t}=N_{0}+C e^{-e^{-B(t-M)}}
$$

Lag phase duration and maximum growth rate were estimated using Eqs. 2 and 3 derived from the Gompertz equation (Zwietering et al. 1990) by substituting the variables $B$ and $M$ calculated from Eq. 1

$$
\begin{gathered}
L=M-\frac{1}{B} \\
\left.\frac{d N_{t}}{d t}\right|_{t=M}=\frac{B C}{e}
\end{gathered}
$$

Since $N_{0}$ is the inoculated dry cell mass and $C$ equals the difference between dry cell mass during steady state and $N_{0}$, the following equation was proposed to calculate the $C$ 
value.

$$
C=C_{\text {Mean }}-N_{0}
$$

The Gompertz $N_{0}$ and $C$ values can be omitted from consideration for model development (Zaika et al. 1992). Therefore, the form of a growth curve is regulated by the values of $B$ and $M$. To predict the growth of organisms from initial storage conditions, the $B$ and $M$ values have to be described in terms of temperature, inoculated organisms and ethanol concentration. Models to calculate the $B$ and $M$ values were constructed by stepwise multiple regression analysis with StatView ${ }^{\circledR}$ ver.4.5 (Hulinks Inc., Tokyo, Japan) of which the dependent variables were the $B$ and $M$ values and the independent variables were the square, cube, reciprocal, reciprocal square, reciprocal cube, exponent, reciprocal exponent and cross product of dry cell mass, ethanol concentration and storage temperature. To calibrate the effectiveness of the generated predictive model, storage experiments were performed at $306 \mathrm{~K}$ using the parameter combinations of inoculated organisms at $2.66 \times 10^{-3} \mathrm{~g} \cdot \mathrm{L}^{-1}$ (Run 1 ) and $2.66 \times 10^{-4} \mathrm{~g} \cdot \mathrm{L}^{-1}$ (Run 2) in dry cell and ethanol concentrations were of 3.5 $\mathrm{g} \cdot \mathrm{L}^{-1}$ (Run 1) and $3.9 \mathrm{~g} \cdot \mathrm{L}^{-1}$ (Run 2), respectively.

Investigation of quality guarantee period Limit of human vision to perceive the presence of the organisms in soup products was examined using the soup samples including dry cell mass ranging from 0 to $0.0281 \mathrm{~g} \cdot \mathrm{L}^{-1}$. This result is expected to contribute to the determination of quality guarantee period of products.

\section{Results}

All the approximated kinetic parameters for the growths of B. amyloliquefaciens KP-991 strain with the Gompertz function were presented in Table 2. The correlative coefficients ranged from 0.985 to 0.999 and were statistically significant at a $99.9 \%$ level of Fisher's ztransformation. Temperature, initial dry cell mass and ethanol concentration dependence of lag phase duration and maximum growth rate expressed by the mean of the growth data obtained from the experiments repeated three times was shown in Fig. 1. The lag phase duration was prolonged by an increase of ethanol and a decrease of temperature and initial cell mass. The temperature dependence of the lag phase duration has also been reported by Dickson et al. (1992) and Bhaduri et al. (1995). The maximum growth rate was accelerated with temperature and initial cell mass. However, the ethanol dependence of the rate was not clearly shown. This is interesting, because no report has been made concerning the influence of ethanol on microbial growth kinetics although the effectiveness of ethanol on the depression of microbial growth has been well documented. The equation for calculating $B$ value in the Gompertz function was generated by stepwise multiple regression analysis as follows:

$$
\begin{aligned}
B & =2.33 \times 10^{7} \boldsymbol{\theta}^{-2}-4.84 \times 10^{9} \boldsymbol{\theta}^{-3}-1574 e^{\zeta} \\
& +5.67 \boldsymbol{\theta} \boldsymbol{\zeta}-25.5 \boldsymbol{\zeta} \boldsymbol{\varepsilon}+1494
\end{aligned}
$$

Since the $C$ value defined by Eq. 4 is fixed in this study, the maximum growth rate is proportional to the $B$ value, according to Eq. 3. Therefore, calculation of $B$ is chiefly composed of the independent variables related to temperature and initial cell mass. Ethanol concentration as an independent variable was implemented in Eq. 5 only as an element of a cross product.The equation for calculating $M$ value in the Gompertz function was generated by the same analysis as Eq. 5, as follows:

$$
\begin{aligned}
M & =3.35 \times 10^{7} \boldsymbol{\zeta}^{3}+0.152 \varepsilon^{3}+1.26 \times 10^{-5} \boldsymbol{\zeta}^{-1} \\
& +2.32 \times 10^{130} e^{-\theta}-487 \zeta \boldsymbol{\varepsilon}+1.16
\end{aligned}
$$

According to the results obtained in this study (data are not presented), $M$ value was well correlated with the lag phase duration $\left(\mathrm{r}=0.957^{* * *}\right.$, significant at a $99.9 \%$ level). Therefore, $M$ value is expected to relate to three kinds of conditions as presented in Eq. 6, as well as the lag phase duration.

Predicted growth calculated by substituting Eqs. 4-6 into Eq. 1 were compared for actual data measured to calibrate the effectiveness of the proposed model on changes in growth of contaminant in a soup product over time (Fig. 2). Since the simulated results agreed well with the experimental data, the proposed model is effective for the prediction of soup spoilage. Perceptible limit of organisms in the soup was 0.195 in O.D. $660 \mathrm{~nm}$, including 0.160 for soup. The net absorbance of 0.035 is equivalent to $0.0139 \mathrm{~g} \cdot \mathrm{L}^{-1}$ dry cell mass according to the calibration curve. Quality guarantee periods of the soup product were calculated as 1.74 (Run 1) and $7.30 \mathrm{~d}$ (Run 2), and the lag phase durations were 2.39 (Run 1) and $8.02 \mathrm{~d}$ (Run 2). A high correlation between quality guarantee period and lag phase duration was observed as shown in Fig. $3\left(\mathrm{r}=0.985^{* * *}\right.$, significant at a $99.9 \%$ level). Since the linear regression equation was $y=0.928 x-0.741$ (Dependent and independent variables are the quality guarantee period and lag phase duration, respectively), the quality guarantee period may be about $18 \mathrm{~h}$ shorter than the lag phase duration.

\section{Discussion}

In many reports, microbial growth data collected as colony formation unit (CFU) has been transformed into logarithm scale for the Gompertz function (Schaffner 1998). On the other hand, we monitored the growths of contaminant by spectrophotometry, which is expected to apply to non destructive evaluation especially effective for liquid products. When the growth data measured by this method is transformed into logarithm scale, extremely precise examination in low ranges of absorbance is needed. No device has been found to carry out such a precise evaluation. Therefore, actual growth data were employed for kinetic analysis in this study.

Absorptivity coefficients of five kinds of genus Bacillus investigated by authors (Makino and Fujisawa 2001) were determined as $0.250-0.298 \mathrm{~m}^{2} \cdot \mathrm{g}^{-1}$ (a $95 \%$ level of confidence interval). The cell sizes of genus Bacillus, aerobic Gram-positive bacilli, are almost constant, even though differences dependent on individual organisms exist. Therefore, the monitoring of genus Bacillus as a main 
Table 2. Effect of storage temperature, ethanol concentration and inoculated dry cell mass on growth kinetics based on the Gompertz function of Bacillus amyloliquefaciens KP-991 strain in a soup product.

\begin{tabular}{|c|c|c|c|c|c|c|c|}
\hline $\begin{array}{l}\text { Storage } \\
\text { temperature, } \\
\mathrm{K}\end{array}$ & $\begin{array}{l}\text { Ethanol } \\
\text { concentration, } \\
\mathrm{g} \cdot \mathrm{L}^{-1}\end{array}$ & $\begin{array}{l}\text { Inoculated } \\
\text { dry cell } \\
\text { mass, } \mathrm{g} \cdot \mathrm{L}^{-1}\end{array}$ & $\begin{array}{l}\text { Difference } \\
\text { between the initial } \\
\text { and maximum dry } \\
\text { cell mass, } \mathrm{g} \cdot \mathrm{L}^{-1}\end{array}$ & $\begin{array}{l}\text { Maximum relative } \\
\text { growth rate }\end{array}$ & $\begin{array}{l}\text { Time at which the } \\
\text { absolute growth } \\
\text { rate is maximum, } \\
\text { d }\end{array}$ & $\begin{array}{l}\text { Calculated initial dry } \\
\text { cell mass, } g \cdot L^{-1}\end{array}$ & $\begin{array}{l}\text { Correlative } \\
\text { coefficient }\end{array}$ \\
\hline \multirow{20}{*}{298} & \multirow{4}{*}{2.7} & $5.32 \times 10^{-3}$ & $0.826 \pm 8.29 \times 10^{-4}$ & $0.228 \pm 1.04 \times 10^{-3}$ & $9.60 \pm 2.71 \times 10^{-2}$ & $2.90 \times 10^{2} \pm 2.69 \times 10^{-4}$ & $0.997 \pm 9.58 \times 10^{-5}$ \\
\hline & & $5.32 \times 10^{-4}$ & $0.944 \pm 2.18 \times 10^{-3}$ & $0.161 \pm 1.55 \times 10^{-3}$ & $12.78 \pm 6.78 \times 10^{-2}$ & $1.34 \times 10^{2} \pm 2.08 \times 10^{-4}$ & $0.994 \pm 2.09 \times 10^{-5}$ \\
\hline & & $5.32 \times 10^{-5}$ & $0.852 \pm 2.92 \times 10^{-3}$ & $0.236 \pm 5.37 \times 10^{-4}$ & $12.15 \pm 1.44 \times 10^{-2}$ & $3.68 \times 10^{2} \pm 3.06 \times 10^{-4}$ & $0.992 \pm 1.46 \times 10^{-4}$ \\
\hline & & $5.32 \times 10^{-6}$ & $0.897 \pm 4.50 \times 10^{-3}$ & $0.198 \pm 3.89 \times 10^{-4}$ & $13.12 \pm 2.97 \times 10^{-2}$ & $1.60 \times 10^{2} \pm 2.62 \times 10^{-4}$ & $0.994 \pm 8.36 \times 10^{-5}$ \\
\hline & \multirow{4}{*}{3.2} & $5.32 \times 10^{-3}$ & $0.882 \pm 1.77 \times 10^{-3}$ & $0.183 \pm 2.94 \times 10^{-4}$ & $10.08 \pm 5.06 \times 10^{-2}$ & $7.20 \times 10^{3} \pm 7.78 \times 10^{-4}$ & $0.996 \pm 4.18 \times 10^{-5}$ \\
\hline & & $5.32 \times 10^{-4}$ & $0.857 \pm 1.57 \times 10^{-3}$ & $0.292 \pm 7.21 \times 10^{-3}$ & $12.05 \pm 6.88 \times 10^{-2}$ & $1.73 \times 10^{2} \pm 1.13 \times 10^{-4}$ & $0.996 \pm 0.00$ \\
\hline & & $5.32 \times 10^{-5}$ & $0.865 \pm 1.58 \times 10^{-3}$ & $0.165 \pm 9.34 \times 10^{-4}$ & $14.82 \pm 4.99 \times 10^{-2}$ & $1.46 \times 10^{2} \pm 3.49 \times 10^{-4}$ & $0.996 \pm 3.62 \times 10^{-5}$ \\
\hline & & $5.32 \times 10^{-6}$ & $0.832 \pm 1.58 \times 10^{-3}$ & $0.193 \pm 2.20 \times 10^{-3}$ & $13.70 \pm 5.49 \times 10^{-2}$ & $1.67 \times 10^{2} \pm 1.57 \times 10^{-4}$ & $0.996 \pm 5.53 \times 10^{-5}$ \\
\hline & \multirow{4}{*}{3.7} & $5.32 \times 10^{-3}$ & $0.856 \pm 1.34 \times 10^{-3}$ & $0.188 \pm 1.32 \times 10^{-3}$ & $13.35 \pm 7.62 \times 10^{-2}$ & $1.59 \times 10^{2} \pm 1.16 \times 10^{-4}$ & $0.996 \pm 7.54 \times 10^{-5}$ \\
\hline & & $5.32 \times 10^{-4}$ & $0.935 \pm 4.96 \times 10^{-4}$ & $0.164 \pm 5.94 \times 10^{-4}$ & $16.89 \pm 1.58 \times 10^{-2}$ & $2.27 \times 10^{2} \pm 2.18 \times 10^{-4}$ & $0.993 \pm 7.24 \times 10^{-5}$ \\
\hline & & $5.32 \times 10^{-5}$ & $0.848 \pm 6.83 \times 10^{-4}$ & $0.175 \pm 3.26 \times 10^{-4}$ & $17.99 \pm 2.97 \times 10^{-2}$ & $2.44 \times 10^{2} \pm 1.10 \times 10^{-4}$ & $0.996 \pm 5.53 \times 10^{-5}$ \\
\hline & & $5.32 \times 10^{-6}$ & $0.920 \pm 3.01 \times 10^{-3}$ & $0.208 \pm 1.25 \times 10^{-3}$ & $25.15 \pm 2.06 \times 10^{-1}$ & $3.19 \times 10^{2} \pm 2.60 \times 10^{-4}$ & $0.991 \pm 1.31 \times 10^{-4}$ \\
\hline & \multirow{4}{*}{4.2} & $5.32 \times 10^{-3}$ & $0.960 \pm 3.47 \times 10^{-3}$ & $0.162 \pm 7.64 \times 10^{-4}$ & $16.46 \pm 7.68 \times 10^{-2}$ & $2.56 \times 10^{2} \pm 4.18 \times 10^{-4}$ & $0.994 \pm 5.53 \times 10^{-5}$ \\
\hline & & $5.32 \times 10^{-4}$ & $0.933 \pm 1.11 \times 10^{-3}$ & $0.158 \pm 1.09 \times 10^{-4}$ & $19.10 \pm 3.51 \times 10^{-2}$ & $2.66 \times 10^{2} \pm 1.05 \times 10^{-4}$ & $0.996 \pm 7.54 \times 10^{-5}$ \\
\hline & & $5.32 \times 10^{-5}$ & $0.889 \pm 1.90 \times 10^{-3}$ & $0.165 \pm 1.04 \times 10^{-3}$ & $18.67 \pm 4.37 \times 10^{-2}$ & $2.22 \times 10^{2} \pm 2.67 \times 10^{-4}$ & $0.997 \pm 8.36 \times 10^{-5}$ \\
\hline & & $5.32 \times 10^{-6}$ & $0.934^{*}$ & $0.166^{*}$ & $18.28^{*}$ & $2.42 \times 10^{2^{2 *}}$ & $0.996^{\dagger}$ \\
\hline & \multirow{4}{*}{4.7} & $5.32 \times 10^{-3}$ & $1.130 \pm 1.64 \times 10^{-2}$ & $0.173 \pm 2.33 \times 10^{-3}$ & $23.01 \pm 1.73 \times 10^{-1}$ & $3.59 \times 10^{2} \pm 1.63 \times 10^{-4}$ & $0.992 \pm 5.53 \times 10^{-5}$ \\
\hline & & $5.32 \times 10^{-4}$ & $1.028 \pm 5.66 \times 10^{-3}$ & $0.143 \pm 2.49 \times 10^{-3}$ & $27.59 \pm 6.81 \times 10^{-2}$ & $2.83 \times 10^{2} \pm 1.74 \times 10^{-4}$ & $0.995 \pm 3.62 \times 10^{-5}$ \\
\hline & & $5.32 \times 10^{-5}$ & $0.972 \pm 3.69 \times 10^{-4}$ & $0.147 \pm 2.68 \times 10^{-4}$ & $28.89 \pm 1.77 \times 10^{-1}$ & $3.00 \times 10^{2} \pm 3.63 \times 10^{-5}$ & $0.998 \pm 2.09 \times 10^{-5}$ \\
\hline & & $5.32 \times 10^{-6}$ & NG & NG & NG & NG & NG \\
\hline \multirow{20}{*}{303} & \multirow{4}{*}{2.7} & $5.32 \times 10^{-3}$ & $0.845 \pm 9.08 \times 10^{-4}$ & $0.671 \pm 2.27 \times 10^{-3}$ & $3.57 \pm 9.18 \times 10^{-3}$ & $1.77 \times 10^{2} \pm 1.45 \times 10^{-4}$ & $0.995 \pm 1.99 \times 10^{-4}$ \\
\hline & & $5.32 \times 10^{-4}$ & $0.812 \pm 2.53 \times 10^{-3}$ & $0.655 \pm 1.85 \times 10^{-2}$ & $5.61 \pm 2.30 \times 10^{-2}$ & $1.27 \times 10^{2} \pm 9.54 \times 10^{-4}$ & $0.998 \pm 2.09 \times 10^{-5}$ \\
\hline & & $5.32 \times 10^{-5}$ & $0.818 \pm 2.23 \times 10^{-4}$ & $0.488 \pm 6.92 \times 10^{-3}$ & $5.60 \pm 2.10 \times 10^{-2}$ & $8.73 \times 10^{3} \pm 5.95 \times 10^{-4}$ & $0.998 \pm 2.09 \times 10^{-5}$ \\
\hline & & $5.32 \times 10^{-6}$ & $0.825 \pm 7.45 \times 10^{-4}$ & $0.332 \pm 2.20 \times 10^{-3}$ & $6.02 \pm 1.99 \times 10^{-2}$ & $5.20 \times 10^{3} \pm 3.37 \times 10^{-4}$ & $0.999 \pm 2.09 \times 10^{-5}$ \\
\hline & \multirow{4}{*}{3.2} & $5.32 \times 10^{-3}$ & $0.821 \pm 3.91 \times 10^{-4}$ & $0.683 \pm 2.73 \times 10^{-3}$ & $3.76 \pm 1.07 \times 10^{-2}$ & $1.25 \times 10^{2} \pm 3.36 \times 10^{-5}$ & $0.999 \pm 53.9 \times 10^{-9}$ \\
\hline & & $5.32 \times 10^{-4}$ & $0.816 \pm 3.80 \times 10^{-4}$ & $0.756 \pm 7.80 \times 10^{-3}$ & $5.08 \pm 1.39 \times 10^{-2}$ & $1.76 \times 10^{2} \pm 3.97 \times 10^{-4}$ & $0.999 \pm 2.09 \times 10^{-5}$ \\
\hline & & $5.32 \times 10^{-5}$ & $0.817 \pm 8.79 \times 10^{-4}$ & $0.376 \pm 3.01 \times 10^{-3}$ & $8.29 \pm 7.28 \times 10^{-2}$ & $7.90 \times 10^{3} \pm 7.88 \times 10^{-4}$ & $0.997 \pm 9.58 \times 10^{-5}$ \\
\hline & & $5.32 \times 10^{-6}$ & $0.800 \pm 1.60 \times 10^{-3}$ & $0.674 \pm 1.30 \times 10^{-2}$ & $9.91 \pm 1.38 \times 10^{-1}$ & $2.73 \times 10^{2} \pm 3.10 \times 10^{-4}$ & $0.998 \pm 5.53 \times 10^{-5}$ \\
\hline & \multirow{4}{*}{3.7} & $5.32 \times 10^{-3}$ & $0.789 \pm 2.07 \times 10^{-3}$ & $0.731 \pm 1.26 \times 10^{-2}$ & $4.60 \pm 1.50 \times 10^{-2}$ & $1.35 \times 10^{2} \pm 4.74 \times 10^{-4}$ & $0.995 \pm 1.11 \times 10^{-4}$ \\
\hline & & $5.32 \times 10^{-4}$ & $0.808 \pm 8.78 \times 10^{-4}$ & $0.432 \pm 4.34 \times 10^{-3}$ & $8.14 \pm 5.48 \times 10^{-2}$ & $1.54 \times 10^{2} \pm 1.79 \times 10^{-4}$ & $0.998 \pm 6.27 \times 10^{-5}$ \\
\hline & & $5.32 \times 10^{-5}$ & $0.796 \pm 4.51 \times 10^{-4}$ & $0.483 \pm 8.51 \times 10^{-3}$ & $9.76 \pm 3.67 \times 10^{-2}$ & $1.86 \times 10^{2} \pm 1.79 \times 10^{-4}$ & $0.998 \pm 4.18 \times 10^{-5}$ \\
\hline & & $5.32 \times 10^{-6}$ & $0.824 \pm 6.01 \times 10^{-4}$ & $0.370 \pm 2.61 \times 10^{-4}$ & $10.05 \pm 4.02 \times 10^{-2}$ & $2.22 \times 10^{2} \pm 1.33 \times 10^{-4}$ & $0.998 \pm 5.53 \times 10^{-5}$ \\
\hline & \multirow{4}{*}{4.2} & $5.32 \times 10^{-3}$ & $0.832 \pm 1.85 \times 10^{-4}$ & $0.552 \pm 1.86 \times 10^{-3}$ & $6.50 \pm 6.85 \times 10^{-3}$ & $1.77 \times 10^{2} \pm 1.86 \times 10^{-5}$ & $0.999 \pm 2.09 \times 10^{-5}$ \\
\hline & & $5.32 \times 10^{-4}$ & $0.839 \pm 6.92 \times 10^{-4}$ & $0.423 \pm 1.66 \times 10^{-3}$ & $8.91 \pm 1.37 \times 10^{-2}$ & $1.82 \times 10^{2} \pm 1.56 \times 10^{-4}$ & $0.997 \pm 5.53 \times 10^{-5}$ \\
\hline & & $5.32 \times 10^{-5}$ & $0.762 \pm 4.96 \times 10^{-3}$ & $0.497 \pm 3.04 \times 10^{-3}$ & $11.33 \pm 5.74 \times 10^{-2}$ & $2.26 \times 10^{2} \pm 1.53 \times 10^{-4}$ & $0.997 \pm 8.36 \times 10^{-5}$ \\
\hline & & $5.32 \times 10^{-6}$ & $0.808 \pm 1.61 \times 10^{-3}$ & $0.594 \pm 1.30 \times 10^{-2}$ & $14.38 \pm 1.36 \times 10^{-1}$ & $2.86 \times 10^{2} \pm 2.06 \times 10^{-4}$ & $0.998 \pm 6.27 \times 10^{-5}$ \\
\hline & \multirow{4}{*}{4.7} & $5.32 \times 10^{-3}$ & $0.814 \pm 1.26 \times 10^{-3}$ & $0.531 \pm 3.33 \times 10^{-3}$ & $9.20 \pm 1.66 \times 10^{-2}$ & $2.54 \times 10^{2} \pm 1.60 \times 10^{-4}$ & $0.998 \pm 2.09 \times 10^{-5}$ \\
\hline & & $5.32 \times 10^{-4}$ & $0.824 \pm 7.59 \times 10^{-4}$ & $0.325 \pm 1.43 \times 10^{-3}$ & $12.62 \pm 1.83 \times 10^{-2}$ & $2.00 \times 10^{2} \pm 1.67 \times 10^{-4}$ & $0.999 \pm 2.09 \times 10^{-5}$ \\
\hline & & $5.32 \times 10^{-5}$ & $0.822 \pm 9.37 \times 10^{-4}$ & $0.343 \pm 1.32 \times 10^{-3}$ & $15.10 \pm 1.19 \times 10^{-1}$ & $2.57 \times 10^{2} \pm 1.34 \times 10^{-4}$ & $0.998 \pm 2.09 \times 10^{-5}$ \\
\hline & & $5.32 \times 10^{-6}$ & $0.837 \pm 3.71 \times 10^{-3}$ & $0.503 \pm 1.04 \times 10^{-2}$ & $23.38 \pm 1.72 \times 10^{-1}$ & $2.93 \times 10^{2} \pm 1.22 \times 10^{-4}$ & $0.998 \pm 8.36 \times 10^{-5}$ \\
\hline \multirow{20}{*}{310} & \multirow{4}{*}{2.7} & $5.32 \times 10^{-3}$ & $0.763 \pm 4.58 \times 10^{-4}$ & $1.717 \pm 7.71 \times 10^{-3}$ & $2.33 \pm 3.00 \times 10^{-3}$ & $1.70 \times 10^{2} \pm 1.11 \times 10^{-5}$ & $0.999 \pm 2.09 \times 10^{-5}$ \\
\hline & & $5.32 \times 10^{-4}$ & $0.838 \pm 7.09 \times 10^{-4}$ & $0.631 \pm 1.19 \times 10^{-2}$ & $4.14 \pm 1.75 \times 10^{-2}$ & $3.20 \times 10^{3} \pm 7.21 \times 10^{-4}$ & $0.994 \pm 1.81 \times 10^{-4}$ \\
\hline & & $5.32 \times 10^{-5}$ & $0.822 \pm 3.37 \times 10^{-3}$ & $0.353 \pm 5.72 \times 10^{-4}$ & $5.78 \pm 1.25 \times 10^{-2}$ & $7.60 \times 10^{3} \pm 4.03 \times 10^{-5}$ & $0.996 \pm 9.11 \times 10^{-5}$ \\
\hline & & $5.32 \times 10^{-6}$ & $0.818 \pm 9.29 \times 10^{-4}$ & $0.426 \pm 3.45 \times 10^{-3}$ & $5.54 \pm 1.96 \times 10^{-2}$ & $1.08 \times 10^{2} \pm 2.66 \times 10^{-4}$ & $0.997 \pm 3.62 \times 10^{-5}$ \\
\hline & \multirow{4}{*}{3.2} & $5.32 \times 10^{-3}$ & $0.888 \pm 2.10 \times 10^{-3}$ & $0.732 \pm 1.56 \times 10^{-2}$ & $2.80 \pm 1.88 \times 10^{-2}$ & $-8.73 \times 10^{3} \pm 7.96 \times 10^{-4}$ & $0.994 \pm 2.83 \times 10^{-4}$ \\
\hline & & $5.32 \times 10^{-4}$ & $0.944 \pm 6.12 \times 10^{-3}$ & $0.540 \pm 2.47 \times 10^{-3}$ & $4.74 \pm 1.81 \times 10^{-2}$ & $1.34 \times 10^{2} \pm 1.24 \times 10^{-4}$ & $0.996 \pm 7.24 \times 10^{-5}$ \\
\hline & & $5.32 \times 10^{-5}$ & $0.810 \pm 1.39 \times 10^{-3}$ & $0.720 \pm 2.49 \times 10^{-2}$ & $7.39 \pm 1.26 \times 10^{-1}$ & $1.34 \times 10^{2} \pm 1.17 \times 10^{-3}$ & $0.996 \pm 7.54 \times 10^{-5}$ \\
\hline & & $5.32 \times 10^{-6}$ & $0.805 \pm 2.26 \times 10^{-4}$ & $0.401 \pm 2.81 \times 10^{-3}$ & $11.03 \pm 2.47 \times 10^{-2}$ & $2.99 \times 10^{2} \pm 1.38 \times 10^{-4}$ & $0.997 \pm 4.18 \times 10^{-5}$ \\
\hline & & $5.32 \times 10^{-3}$ & $0.874 \pm 3.57 \times 10^{-3}$ & $0.688 \pm 1.50 \times 10^{-2}$ & $4.33 \pm 1.97 \times 10^{-2}$ & $9.37 \times 10^{3} \pm 3.31 \times 10^{-4}$ & $0.998 \pm 2.09 \times 10^{-5}$ \\
\hline & & $5.32 \times 10^{-4}$ & $0.812 \pm 3.67 \times 10^{-4}$ & $0.546 \pm 8.74 \times 10^{-3}$ & $6.76 \pm 7.55 \times 10^{-2}$ & $1.39 \times 10^{2} \pm 5.63 \times 10^{-4}$ & $0.999 \pm 53.9 \times 10^{-9}$ \\
\hline & 3.7 & $5.32 \times 10^{-5}$ & $0.794 \pm 1.64 \times 10^{-3}$ & $0.842 \pm 1.39 \times 10^{-2}$ & $9.79 \pm 1.41 \times 10^{-1}$ & $3.17 \times 10^{2} \pm 1.46 \times 10^{-4}$ & $0.998 \pm 53.9 \times 10^{-9}$ \\
\hline & & $5.32 \times 10^{-6}$ & $0.901 \pm 5.01 \times 10^{-3}$ & $0.941 \pm 4.92 \times 10^{-3}$ & $11.16 \pm 1.78 \times 10^{-1}$ & $3.62 \times 10^{2} \pm 2.00 \times 10^{-4}$ & $0.998 \pm 3.62 \times 10^{-5}$ \\
\hline & & $5.32 \times 10^{-3}$ & $0.888 \pm 3.98 \times 10^{-3}$ & $1.011 \pm 1.99 \times 10^{-2}$ & $4.78 \pm 3.13 \times 10^{-2}$ & $3.08 \times 10^{2} \pm 2.90 \times 10^{-4}$ & $0.995 \pm 1.46 \times 10^{-4}$ \\
\hline & & $5.32 \times 10^{-4}$ & $0.796 \pm 1.04 \times 10^{-3}$ & $0.824 \pm 1.02 \times 10^{-2}$ & $12.31 \pm 2.65 \times 10^{-1}$ & $2.94 \times 10^{2} \pm 3.95 \times 10^{-4}$ & $0.999 \pm 2.09 \times 10^{-5}$ \\
\hline & 4.2 & $5.32 \times 10^{-5}$ & $0.849 \pm 2.79 \times 10^{-3}$ & $0.744 \pm 7.80 \times 10^{-3}$ & $12.83 \pm 2.18 \times 10^{-1}$ & $3.34 \times 10^{2} \pm 1.45 \times 10^{-4}$ & $0.998 \pm 5.53 \times 10^{-5}$ \\
\hline & & $5.32 \times 10^{-6}$ & $0.793 \pm 1.60 \times 10^{-3}$ & $0.581 \pm 3.32 \times 10^{-3}$ & $12.85 \pm 1.50 \times 10^{-1}$ & $3.22 \times 10^{2} \pm 2.21 \times 10^{-4}$ & $0.999 \pm 2.09 \times 10^{-5}$ \\
\hline & & $5.32 \times 10^{-3}$ & $0.877 \pm 2.73 \times 10^{-3}$ & $0.821 \pm 9.75 \times 10^{-3}$ & $7.17 \pm 9.96 \times 10^{-3}$ & $2.61 \times 10^{2} \pm 1.12 \times 10^{-4}$ & $0.998 \pm 2.09 \times 10^{-5}$ \\
\hline & & $5.32 \times 10^{-4}$ & $0.788 \pm 1.13 \times 10^{-3}$ & $1.072 \pm 1.99 \times 10^{-2}$ & $16.31 \pm 2.89 \times 10^{-1}$ & $3.78 \times 10^{2} \pm 1.71 \times 10^{-4}$ & $0.997 \pm 4.18 \times 10^{-5}$ \\
\hline & 4.7 & $5.32 \times 10^{-5}$ & $0.774 \pm 3.67 \times 10^{-4}$ & $0.687 \pm 8.91 \times 10^{-3}$ & $16.25 \pm 1.51 \times 10^{-1}$ & $3.58 \times 10^{2} \pm 2.77 \times 10^{-4}$ & $0.999 \pm 2.09 \times 10^{-5}$ \\
\hline & & $5.32 \times 10^{-6}$ & $0.783 \pm 1.15 \times 10^{-3}$ & $0.490 \pm 3.05 \times 10^{-3}$ & $18.60 \pm 1.09 \times 10^{-1}$ & $3.40 \times 10^{2} \pm 1.22 \times 10^{-4}$ & $0.999 \pm 2.09 \times 10^{-5}$ \\
\hline
\end{tabular}

Individual data are the means of three replicates \pm confidence interval at a $95 \%$ significance.

${ }^{*}$ No confidence interval due to the deficiency of experimental data, NG: No Growth 
contaminant of processed soy sauces can be performed by the same method as proposed in this study.

Kinetic analysis of contaminant growths in the soup product supported the effectiveness of ethanol for prolonging the lag phase duration (Fig. 1) in relation to the quality guarantee period from a predictive microbiological viewpoint. Therefore, ethanol can be applied to prolong the quality guarantee period unless the taste of the product is affected. Ethanol is not useful for slowing the maximum growth rate. This observation is not related to sustainability of quality because the time $M$ is later by $1 / B \mathrm{~d}$ than the lag phase duration; that is, it is after quality guarantee period. Ethanol may have a similar effect with the reduction of initial cell mass because it is effective for killing organisms or halting metabolism in organisms which is different from the effectiveness of decreasing temperature for the depression of metabolic activities. However, the difference of influence on the maximum growth rate between the addition of ethanol and decrease of initial cell mass was observed. This is an interesting viewpoint for predictive food microbiology.

The multiple regression analysis employed in this study, implements the interactions between storage conditions, is similar to response surface analysis using quadratic equation. Temperature dependence of microbial growth has frequently been expressed using square-root models for which temperature is only a variable condition (Ratkowsky et al. 1982, 1983) or $\mathrm{NaCl}$ concentration and water activity as variables were implemented in the model (McMeekin et al. 1992). A square-root model may be proposed in this study. However, the model equation may have a considerably more complex form because the coefficients and constants have to be defined as functions of storage conditions. Models based on fundamental
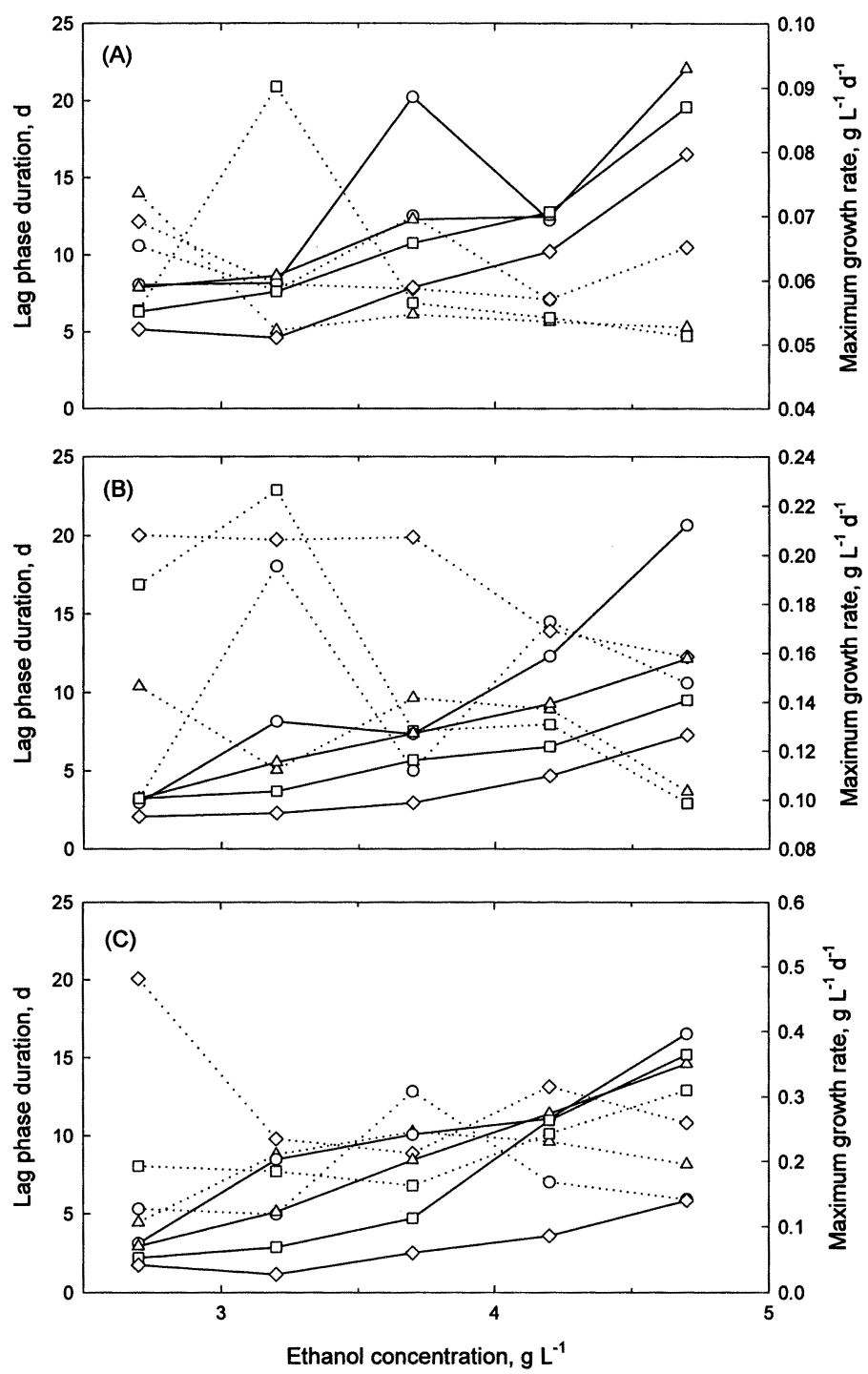

Fig. 1. Changes in lag phase duration (solid line) and maximum growth rate (dashed line) of Bacillus amyloliquefaciens KP-991 strain in a soup product at (A) 278, (B) 303 or (C) $310 \mathrm{~K}$ with ethanol concentration. Circle, triangle, square or diamond denotes $5.32 \times 10^{-6}, 5.32 \times 10^{-6}, 5.32 \times 10^{-6}$ or $5.32 \times 10^{-6} \mathrm{~g}$. $\mathrm{L}^{-1}$ initial dry cell mass, respectively. All the data were obtained from the experiments repeated three times. 


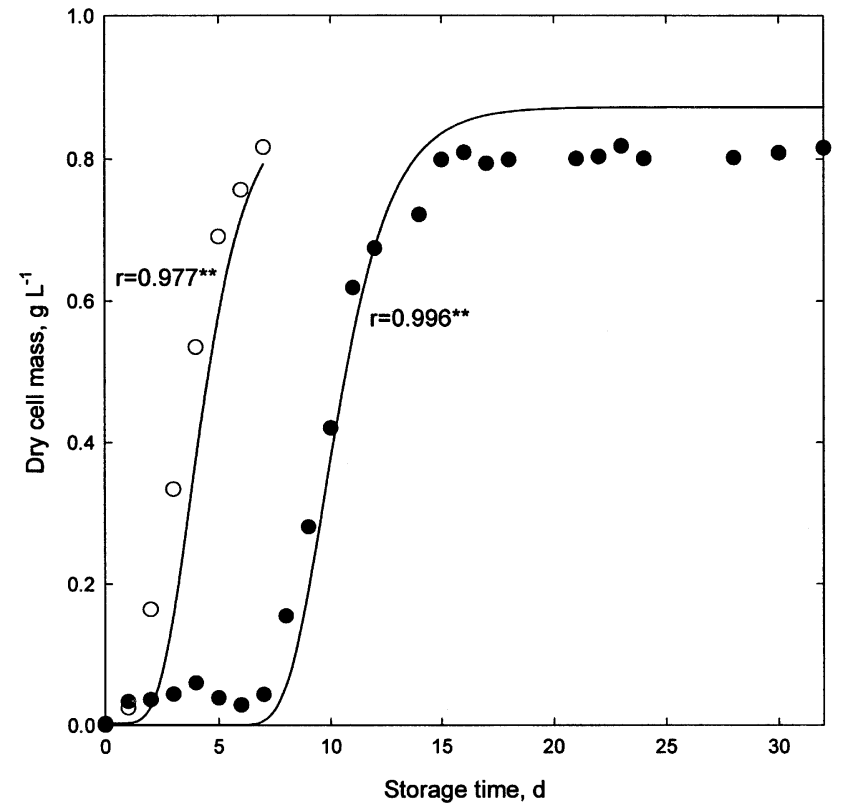

Fig. 2. Actual (symbols) and predicted (solid lines) growths of Bacillus amyloliquefaciens KP-991 strain in a soup product over time at $306 \mathrm{~K}$.

Open (Run 1) or solid (Run 2) circle denotes the microbial growth under the parameter combinations of inoculated organisms $2.66 \times 10^{-3} \mathrm{~g} \cdot \mathrm{L}^{-1}$ (Run 1) and $2.66 \times 10^{-4} \mathrm{~g} \cdot \mathrm{L}^{-1}$ (Run 2) in dry cell and ethanol concentrations $3.5 \mathrm{~g} \cdot \mathrm{L}^{-1}$ (Run 1) and $3.9 \mathrm{~g} \cdot \mathrm{L}^{-1}$ (Run 2), respectively.

principles may also suggest additional control measures useful in extending shelf life or improving food safety. Models that more closely reflect the real uncertainty in the data are also more helpful in risk assessment used to set policy compared to models that oversimplify uncertainty patterns.

\section{Notations}

$B \quad$ Maximum relative growth rate (relative growth rate at $M$ )

$C$ Difference between the initial and maximum dry cell mass, $\mathrm{g} \cdot \mathrm{L}^{-1}$

e Naperian base

$L \quad$ Lag phase duration, d

$M \quad$ Time at which the absolute growth rate is maximum, d

$N \quad$ Dry cell mass, $\mathrm{g} \cdot \mathrm{L}^{-1}$

$r \quad$ Correlative coefficient

$t \quad$ Storage time, $\mathrm{d}$

$x$ an independent variable of an equation, $\mathrm{d}$

$y \quad$ a dependent variable of an equation, $\mathrm{d}$

$\varepsilon \quad$ Ethanol concentration, $\mathrm{g} \cdot \mathrm{L}^{-1}$

$\theta \quad$ Storage temperature, $\mathrm{K}$

$\zeta \quad$ Inoculated dry cell mass, $\mathrm{g} \cdot \mathrm{L}^{-1}$

Subscript

Mean Grand mean of experimental data

$t \quad$ Arbitrary time

$0 \quad$ Initial

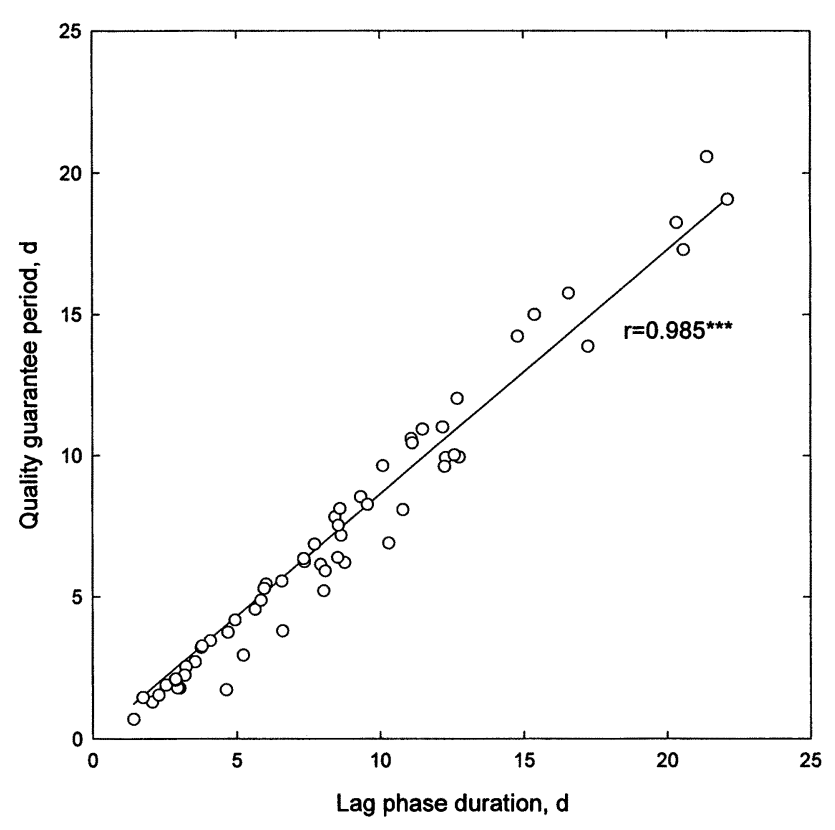

Fig. 3. Relationship between lag phase duration of Bacillus amyloliquefaciens KP-991 strain growth and quality guarantee period of a soup product of which the symbols and solid line denote the observed kinetic data and linear regression line $(y=0.928 x-0.741$, Dependent and independent variables are the quality guarantee period and lag phase duration, respectively).

\section{References}

Bhaduri, S., Buchanan, R.L. and Phillips J.G. (1995). Expanded response surface model for predicting the effects of temperatures, $\mathrm{pH}$, sodium chloride contents and sodium nitrite concentrations on the growth rate of Yersinia enterocolitica. J. Appl. Bacteriol., 79, 163-170.

Buchanan, R.L. and Phillips, J.G. (1990). Response surface model for predicting the effects of temperature, $\mathrm{pH}$, sodium chloride content, sodium nitrite concentration and atmosphere on the growth of Listeria monocytogenes. J. Food Protection, 53, 370376.

Claus, D. and Berkeley, R.C.W. (1986). Genus Bacillus. In "Bergey's Manual of Systematic Bacteriology Vol. 2", ed. by P.H.A. Sneath. Williams \& Wilkins, Baltimore, pp. 1105-1139.

Dickson, J.S., Siragusa, G.R. and Wray Jr., J.E. (1992). Predicting the growth of Salmonella typhimurium on beef by using the temperature function integration technique. Appl. Environ. Microbiol., 58, 3482-3487.

Haga, H. and Endo, K. (1971). On the bacteria and the sporeforming bacteria of shoyu. Seasoning Sci., 18, 14-20.

Makino, Y. and Fujisawa, H. (2001). Characteristics of Bacillus amyloliquefaciens isolated from soy sauce and influence on putrefaction of processed soy sauce with less salt. Jpn. J. Food Eng., 2, 147-152.

McClure, P.J., Cole, M.B. and Davies K.W. (1994). An example of the stages in the development of a predictive mathematical model for microbial growth: the effects of $\mathrm{NaCl}, \mathrm{pH}$ and temperature on the growth of Aeromonas hydrophila. Int. J. Food Microbiol., 23, 359-375.

McMeekin, T.A., Ross, T. and Olley, J. (1992). Application of predictive microbiology to assure the quality and safety of fish and fish products. Int. J. Food Microbiol., 15, 13-32.

Ohtomi, A., Kitakura Y., Someya, S. and Hashimoto, H. (1992). Effects of microorganisms during aging on quality of "Kaeshi" 
for "Sobatsuyu", Japanese noodle soup. Nippon Shokuhin Kogyo Gakkaishi, 39, 1011-1015

Ratkowsky, D.A., Lowry, R.K., McMeekin, T.A., Stokes, A.N. and Chandler, R.E. (1983). Model for bacterial culture growth rate throughout the entire biokinetic temperature range. $J$. Bacteriol., 154, 1222-1226.

Ratkowsky, D.A., Olley, J., McMeekin, T.A. and Ball, A. (1982). Relationship between temperature and growth rate of bacterial cultures. J. Bacteriol., 149, 1-5.

Ross, T. and McMeekin, T.A. (1994). Predictive microbiology. Int. J. Food Microbiol., 23, 241-264.

Schaffner, D.W. (1998). Predictive food microbiology gedanken experiment: why do microbial growth data require a transformation. Food Microbiol., 15, 185-189.
Shoolfield, R.M., Sharpe, P.J.H. and Magnuson, C.E. (1981). Nonlinear regression of biological temperature-dependent rate models based on absolute reaction-rate theory. J. Theor. Biol., 88, 719-731.

Terasawa, M., Matsuwaka, A., Yamashita, K. and Mori, Y. (1993). Effect of temperature on sterilization of "Tsuyu". J. Japan Soy Sauce Research Institute, 19, 71-74.

Zaika, L.L., Phillips, J.G. and Buchanan, R.L. (1992). Model for aerobic growth of Shigella flexneri under various conditions of temperature, PH, sodium chloride and sodium nitrite concentrations. J. Food Protection, 55, 509-513.

Zwietering, M.H., Jongenburger, I., Rombouts, F.M. and Van't Riet, K. (1990). Modeling of the bacterial growth curve. Appl. Environ. Microbiol., 56, 1875-1881. 